\title{
SYNTHESIS OF POLYDIMETHYLSILOXANE MICROSPHERES USING MICROFLUIDICS FOR TREATMENT OF TOLUENE IN WASTEWATER
}

\author{
Zheng Lian ${ }^{\mathrm{a}, \mathrm{b}}$, Yong Ren ${ }^{\mathrm{a}, \mathrm{b}^{*}}$, Jun $\mathrm{He}^{\mathrm{a}, \mathrm{c}}$, George Z. Chen ${ }^{\mathrm{c}}$, and Kai Seng Koh ${ }^{\mathrm{d}}$ \\ ${ }^{a}$ International Doctoral Innovation Centre, University of Nottingham Ningbo China, Ningbo, China \\ ${ }^{\mathrm{b}}$ Department of Mechanical, Materials \& Manufacturing Engineering, University of Nottingham Ningbo China, Ningbo, China \\ c Department of Chemical and Environmental Engineering, University of Nottingham Ningbo China, Ningbo, China
}

${ }^{\mathrm{d}}$ School of Engineering and Physical Sciences, Heriot-Watt University Malaysia, No.1 Jalan Venna P5/2, Precinct 562200

Putrajaya Malaysia

*Correspondence: yong.ren@nottingham.edu.cn; Tel.: +86-574-88180000-9064

\begin{abstract}
Monodispersed polydimethylsiloxane (PDMS) microspheres are fabricated by a needle-based versatile microfluidic device with flow-focusing geometry. Microdroplets with various diameters are generated by tuning the flow rate of the dispersed and continuous phase, using single emulsion as the template. The collected PDMS microdroplets are then thermally cured to form solid microspheres. We employ an optical microscope to observe the particles and capture photos. The figures are processed by ImageJ which measures the diameter of each particle. The coefficients of variation $(\mathrm{CV})$ of particles are found to be less than $1.5 \%$ for all sizes, indicating that a high monodispersity has been achieved. Thereafter, we adopt the PDMS microspheres to a simulated industrial wastewater that contains organic pollutant such as toluene, for removing purposes. Since the solubility parameters of PDMS and toluene are close, toluene molecules can be extracted from its solvent into PDMS. The absorption efficiency provided by PDMS
\end{abstract}

microspheres on organic pollutant such as toluene has been tested by utilizing a Headspace-Gas Chromatography (GC-HS). We then compare the obtained peak signals of toluene before and after treatment to verify the treatment effect. The needle-based microfluidic device is advantageous in its facile assembly and low cost, displaying a great potential for industrial applications.

\section{INTRODUCTION}

As the industrialization and rapid population growth worldwide in recent decades, wastewater treatment has become a critical challenge. Pollutants contained in the wastewater such as toluene and phenol, could pose harms not only to the environment but also exhibit great threads to public health ${ }^{1}$. Hence, it is a vital issue to remove such pollutants in wastewater, in regard to ensure public health and realize sustainable development in the aspects of saving resources and environmental conservation. Conventional 
wastewater treatment approaches mostly focus on large treatment systems which are chemically, energetically and operationally intensive so that occupying considerable infusion of capital, engineering expertise and infrastructure, all of which precludes their uses in many regions ${ }^{2}$. For instance, the use of chemical coagulation and activated sludge suffer great limit of operation costs. This situation also happened in the application of activated carbon $^{3}$. Moreover, water contamination could be exacerbated under the conditions of chemical residuals, such as chlorine compounds, hydrochloric acid, sodium hydroxide, and corrosion control chemicals ${ }^{2}$. Novel methods to fabricate treatment materials applied to pollutant treatment of wastewater were developed in recent years. Miao and Wang utilized starch microspheres by reversed phrase suspension method to treat toluene in wastewater and achieved a maximum absorption as $0.08 \mathrm{mmol} / \mathrm{g}^{4}$. This research inspired the application of microspheres to wastewater treatment. However, the investigation did not explore the removal efficiency of toluene in details. A simple in situ method to generate AuNP/PDMS foam could realise $42 \%$ removal efficiency (from $100 \mathrm{ppm}$ to $58 \mathrm{ppm}$ ) of toluene in wastewater, by Scott et $\mathrm{al}^{5}$. Although the removal efficiency was acceptable, the application involved the use of gold which was not cost-effective for bulk production and thus affect the industrial application.

Microfluidics technologies which consume small amount of fluids and materials while dealing with targets efficiently, provides new opportunities and approaches on facing the challenges ${ }^{6}$. Microfluidic systems facilitate the reduction both on the amounts of reagents used and residues left. Since it is a small portable system with hundreds of micrometres-scales of channel sizes, the total volume of the device is significantly smaller than traditional large systems. In addition, the analyzing speed is enhanced attributed to the fast mixing of reagents inside the channels and thus more complete reactions can be achieved ${ }^{7}$. Because the channel is small, the generated droplets are with high specific surface area, which provides great potential for numerous applications. One of these applications is chemical synthesis for functional materials, which offers unique alternative solutions to waste water treatment, other than conventional approaches.

PDMS owns certain degree of compatibility with organic solvents into which it can swell when submersed. Hildebrand value or $\delta_{\mathrm{H}}$, also defined as solubility parameter is corelated with the capability of one substance dissolving in another, and it is an expression of cohesive energy density ${ }^{8}$. Similar solubility parameters (or cohesive energy densities) are crucial for two materials to be soluble since inserting solvents would require conquering the cohesive energy between molecules. Therefore, organic solvents with similar cohesive energy density with PDMS will swell it effectively. In reverse, the swelling effect of PDMS might be employed for extracting organic chemicals from solvents. Toluene with a solubility parameter of 8.9 $\left(\mathrm{cal}^{1 / 2} \mathrm{~cm}^{-3 / 2}\right.$ ) which is close to that of PDMS 7.3 $\left(\mathrm{cal}^{1 / 2} \mathrm{~cm}^{-3 / 2}\right)$ and thus can be regarded as high solubility $^{9}$. It was noted that PDMS could recover back into its original shapes when the absorbed solvents volatilised from PDMS or transferring the swelled polymers into a lower soluble solvent ${ }^{10}$.

This may propose possible applications for recycling and reuse of PDMS materials. Koh et al. carried out researches on PDMS swelling effect by weight loss method which achieved similar experiment outcomes ${ }^{11}$. 
To the best of our knowledge, it has not been reported that PDMS microparticles fabricated by microfluidic approach adopted for organic pollutant treatment such as toluene absorption in wastewater. We implemented Oil-in-Water $(\mathrm{O} / \mathrm{W})$ single emulsion as template to from PDMS single emulsion microdroplet by microfluidics. Analytical measurement by GC-Headspace is conducted to investigate the absorption results. Despite of toluene, PDMS could be utilized to deal with a number of organic compounds that have similar solubility parameters as PDMS, such as hexane, acetate and chloroform ${ }^{11}$. The needle-based microfluidic device was easily assembled and can be cleaned and reused several times ${ }^{12}$. This device was much more cost-effective than conventional microfluidic device such as glass-or PDMS-based. Hence, parallel connecting could be realized to scale-up the yields, and thus it occupied great potential for industrial application.

\section{EXPERIMENTAL}

\section{MATERIALS AND INSTRUMENTS}

Sylgard

184silicone

elastomer,

Polydimethylsiloxane (PDMS) purchased from Dow Corning (USA) was prepared as dispersed phase liquid with a mass ratio of 10:2 for monomer and curing agent. Polyvinyl Alcohol (PVA) purchased from Sigma Aldrich (Shanghai, China) was mixed with DI water to obtain aqueous solution with a concentration of $5.0 \mathrm{wt} \%$. The PVA aqueous solution was served as continuous phase and surfactant to stabilize the liquid.

Microfluidic device employed in this research was a glue-free needle-based microfluidic device, as demonstrated in fig. 1. A dispensing needle which is defined as inlet needle was connected to a polypropylene (PP) cross-shaped link by a length of silicone rubber soft tube. Another needle (defined as outlet needle), whose needle holder was removed, was inserted into the other tip of the cross-link. The distance between those two needles was fixed as $300 \mu \mathrm{m}$. In this fashion, the tubes of needles and the interspace between the needles structured as microchannels which the diameters could varied according to the selected needle sizes. In this research, we employed $21 \mathrm{G}$ (i.d. $=510 \mu \mathrm{m}$, o.d. $=800 \mu \mathrm{m}$ ) and $20 \mathrm{G}$ (i.d. $=600 \mu \mathrm{m}$, o.d. $=900 \mu \mathrm{m}$ ) needles as inlet and outlet needles, respectively. The above-mentioned materials were purchased from online market Alibaba. Fluids were constantly fed into the microchannel from three $10 \mathrm{ml}$ syringes where were mounted onto two syringe pumps (Longer LSP02-1B).

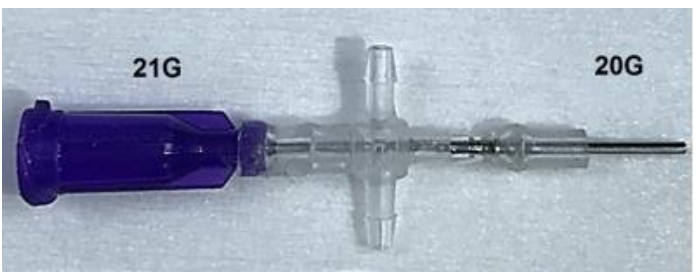

Fig. 1 Glue-free needle-based microfluidic devices for single emulsion microdroplet fabrication

\section{FABRICATION OF PDMS MICRODROPLETS AND MICROSPHERES}

The PDMS pre-mixer was adequately stirred and degassed to remove air bubbles in the liquid body. Then it was injected into the microfluidic system along the inlet needle under a pre-set flow rate. Meanwhile, PVA aqueous solution was introduced into the microchannels from the other two inlets of the link that perpendicular to the needle direction. The two immiscible fluids encountered at the tip of needle and, subsequently the PDMS stream broke up into droplets under the competition of viscous shear stress and interfacial tension between these fluids ${ }^{13}$.The outlet needle acted as an orifice which restricted the continuous phase flow direction, leading to a flow-focusing geometry inside the device. Additionally, the symmetric structure could effectively avoid the impact of wettability on the channel walls and surface modification upon the 
channels walls turned out to be unnecessary ${ }^{14}$. Flow rates of the dispersed and continuous phase fluids were tuned from the syringe pumps. PDMS droplets were formed in dripping regime which a monodispersity was expected ${ }^{15}$. Microdroplets were collected into a beaker that contained water and then thermally cured under $70^{\circ} \mathrm{C}$ for $2 \mathrm{~h}$. The solidified PDMS microspheres were washed by DI water three times and carefully collected into small plastic bottles for storage.

\section{CHARACTERIZATION}

An optical microscope (N-800F, Ningbo Novel Optics, China) was used to observe morphology of the resulting droplets and the cured solids. Images were captured by a connecting desktop computer and processed by a software ImageJ, which was capable to measure the sizes of each droplet or sphere. One of the important parameters to describe the particle size monodispersity is the coefficient of variation $(\mathrm{CV})$, which can be calculated based on the measured standard deviation (STD) and average diameter $(\mathrm{AD})$ of particles, based on the following equation:

$\mathrm{CV}(\%)=\frac{S T D}{A D} \times 100 \%$

\section{ORGANIC POLLUTANT TREATMENT BY PDMS MICROSPHERES}

Toluene solution purchased from Ourchem $(99.8 \%$, Sinopharm Chemical Reagent Co., Ltd) was diluted into $350 \mathrm{ppm}$ which acted as the simulated wastewater. Concentration of such sample was measured after absorption by PDMS. Toluene standard sample was bought from Aladdin (Shanghai, China). Agilent7890B Gas-chromatograph (GC) connected with Agilent7697 Headspace Sampler (HS) was utilised for testing the PDMS treatment results of the organic substances. The toluene removal efficiency $\eta$ was calculated based on the concentration of untreated wastewater $\mathrm{C}_{0}$ and that after treatment $\mathrm{C}_{\mathrm{t}}$. It could be worked out by the following equation:
$\eta=\frac{C 0-C t}{C 0} \times 100 \%$

\section{RESULTS AND DISCUSSION FABRICATION OF PDMS MICROSPHERES}

Solid PDMS microspheres were obtained from the fabricated microdroplets after two-hours thermal curing. After solidification, the solid microspheres were randomly picked from the batch and measured by using software ImageJ. Fig. 2 depicted the solidified PDMS microspheres under microscope that the flow rate combination for disperse phase $Q_{d}$ $=15 \mu \mathrm{l} / \mathrm{min}$ and the continuous phase $\mathrm{Q}_{\mathrm{c}}=$ $200 \mu \mathrm{l} / \mathrm{min}$, respectively. The average diameter of the microparticles was $647 \mu \mathrm{m}$ with coefficient of variation $(\mathrm{CV})$ of $0.67 \%$ from equation (1), which implies a pretty high monodispersity in terms of the particle size. A standard distribution regarding the particle size was depicted in Fig. 3, which revealed most of the diameter values converged at the average value. Hence the average value of diameters might be used to calculate the number of particles required for each treatment, if knowing total amount of the organic targets in advance, and estimate the working efficiency of each single piece of particle. The average production rate of such spheres was recorded as every 30 pieces in 22.5 seconds which made it possible as 4800 pieces per hour.

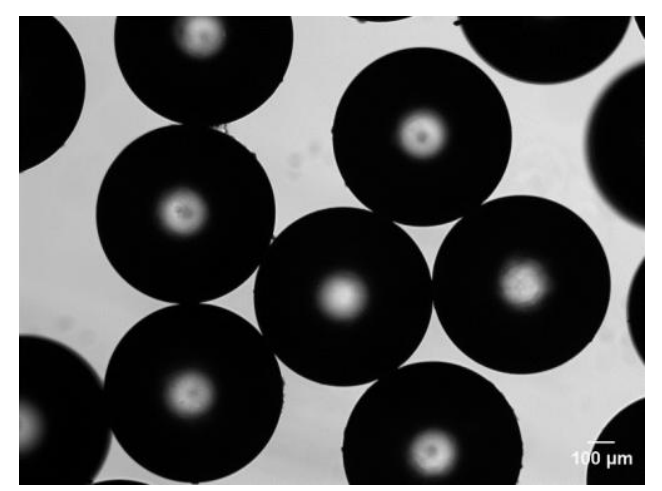

Fig. 2 An optical microscope photograph of PDMS microspheres thermally cured from the templated PDMS microdroplets with flow rates of the disperse 
phase $\mathrm{Q}_{\mathrm{d}}=15 \mu \mathrm{L} / \mathrm{min}$ and the continuous phase $\mathrm{Q}_{\mathrm{c}}=$ $200 \mu \mathrm{l} / \mathrm{min}$, respectively. The scale bar is $100 \mu \mathrm{m}$.

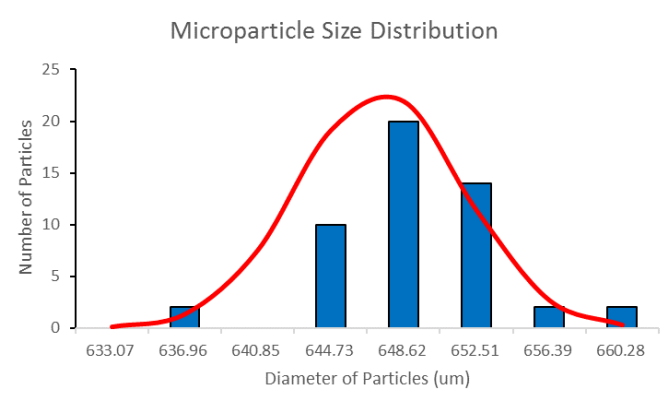

Fig. 3 The size distribution of the collected PDMS microparticles obeyed a standard distribution.

In terms of harvesting PDMS microspheres with various sizes, diverse flow rate combinations regarding the dispersed and continuous phase were accomplished. Due to high viscosity of PDMS (2244cSt), small flow rate exerted at PVA aqueous solution might lead to parallel flow which failed to pinch off the PDMS stream to form droplets or plug

flow in squeezing regime ${ }^{16}$. Whereas high flow rate of the continuous phase could transport PDMS fluids further downstream and pinched off at an elongated tip (known as jetting), resulting polydispersity of the droplets ${ }^{16}$. To ensure the droplets generated in dripping regime, we confined the maximum and minimum flow rates of the continuous phase as $100 \mu \mathrm{l} / \mathrm{min}$ and $300 \mu \mathrm{l} / \mathrm{min}$, respectively, shown in Table. 1. Fixing the dispersed flow rate and increasing the continuous flow resulted smaller microspheres which Fig.4 exemplified this tendency. On the other hand, as listed in Table. 2, fixing the continuous phase as $100 \mu \mathrm{l} / \mathrm{min}$ and increasing flow rates of PDMS dispersed phase, an increasing of microparticle diameter was observed (following in Fig. 5). The experimental results coupled well with the fact that higher continuous flow rate and smaller dispersed phase flow rates would induce smaller diameters of the microspheres ${ }^{17}$. Notably, particle size coefficient of variations (CV) of all groups were below $1.5 \%$ which indicated high monodispersed microparticles were fabricated under corresponding flow rates. Larger range of particle sizes could be obtained by adjusting the inter-needle distance between the inlet and outlet needles or select other needles with diverse diameters.

Table. 1 Diameters of PDMS microspheres collected under diverse flow rates, fixing flow rate of dispersed phase as $15(\mu \mathrm{l} / \mathrm{min})$.

\begin{tabular}{ccc}
\hline $\begin{array}{c}\text { Flow rates } \\
(\mu \mathrm{l} / \mathrm{min})\end{array}$ & $\begin{array}{c}\text { Diameter } \\
(\mu \mathrm{m})\end{array}$ & $\mathrm{CV}(\%)$ \\
\hline $15-100$ & 678.95 & 1.15 \\
$15-150$ & 669.70 & 1.06 \\
$15-200$ & 647.35 & 0.67 \\
$15-250$ & 633.09 & 0.68 \\
$15-300$ & 618.46 & 0.37 \\
\hline
\end{tabular}

$\square 15-\mathrm{x}$

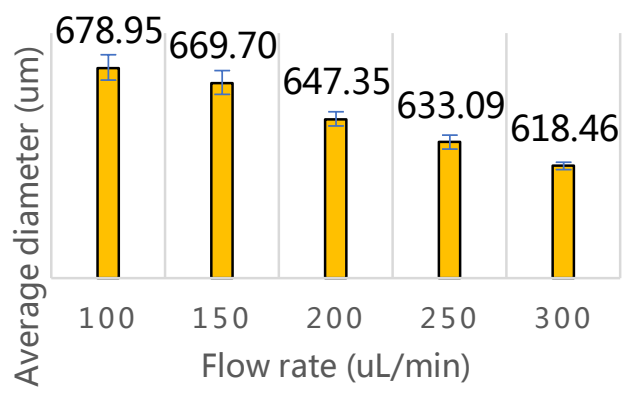

Fig. 4 Diameters of PDMS microspheres collected under diverse flow rates, fixing flow rate of dispersed phase as $15(\mu 1 / \mathrm{min})$.

Table. 2 Diameters of PDMS microspheres collected under diverse flow rates, fixing flow rate of continuous phase.

\begin{tabular}{ccc}
\hline $\begin{array}{c}\text { Flow } \\
\text { rates } \\
(\mu 1 / \mathrm{min})\end{array}$ & $\begin{array}{c}\text { Diameter } \\
(\mu \mathrm{m})\end{array}$ & CV $(\%)$ \\
\hline $10-100$ & 666.94 & 0.78 \\
$15-100$ & 678.95 & 1.15 \\
$20-100$ & 693.87 & 0.47 \\
$30-100$ & 742.38 & 1.31 \\
\hline
\end{tabular}


$\square x-100$

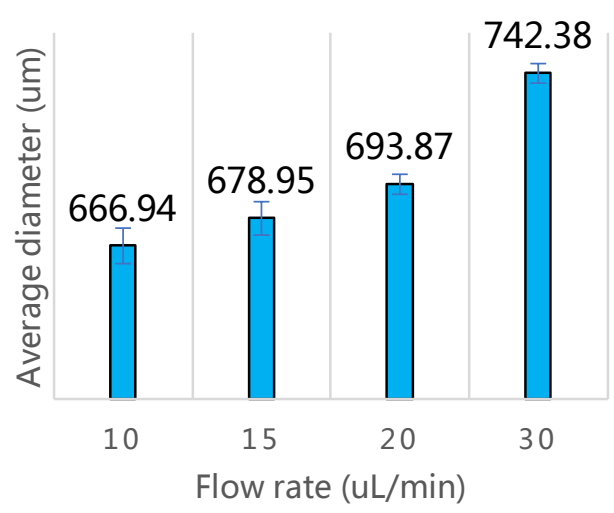

Fig. 5 Diameters of PDMS microspheres collected under diverse flow rates, fixing flow rate of continuous phase.

\section{ABSORPTION OF TOLUENE IN SIMULATED WASTEWATER BY THE FABRICATED PDMS MICROSPHERES}

An array of 20 pieces PDMS microspheres that weighted $4.4 \mathrm{mg}$ in total were released into $15 \mathrm{~mL}$ of a simulated organic wastewater containing 350 ppm toluene. Another toluene sample at the same concentration without treatment was also tested as a control trail. The vials were subsequently sealed and stored. We observed that the microspheres were floating at the liquid surface due to density difference. This phenomenon was beneficial for the treatment, since toluene was slightly dissolved in water, a part of which that floating at the liquid surface could be absorbed into the sphere bodies. The swollen spheres then gradually submersed and precipitated to the bottom. During this process more toluene were captured. The vial was sealed for $24 \mathrm{~h}$ allowing a complete absorption of the organic compounds which saturation of those spheres could be reached. Later $9 \mathrm{ml}$ of the liquids were transferred into headspace vials for testing. The equilibrium temperature of the samples was set as $70^{\circ} \mathrm{C}$ from GC-Headspace panel and equilibrium time was 40min. Fig. 6 depicted peak signal of toluene tested by GC-HS, where the retention time was $3.6 \mathrm{~min}$. The system could integrate the peak area which was transferred to concentration according to the sample calibration curve. The toluene standard sample calibration curve was produced based on toluene concentration gradient as 1, 10, 100, 200 and $400 \mathrm{ppm}$, respectively (refer to inset of Fig. 6). The correlation coefficient $r$ was found to be 0.9991 for calibration curve formula: $y=61.09 x-159.24$, where $x$ represented the amount of toluene (ppm) and y was the area of toluene peak. The measured concentration before treatment was 340 ppm which also validated that a reliable calibration curve was capable to be implemented for analytical measurement.

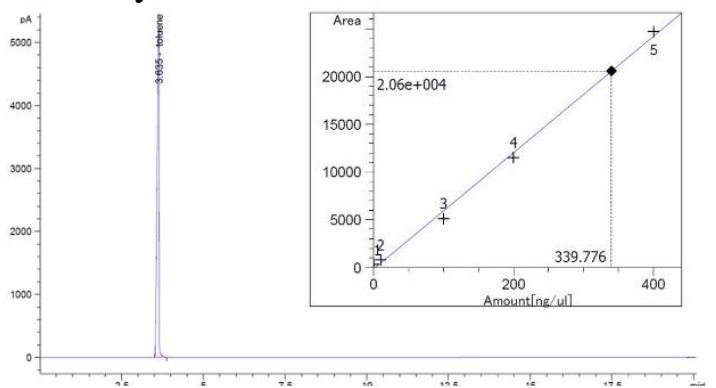

Fig. 6 Toluene peak signal before treatment

As the sole simulated pollutant added into the liquid is toluene, the peak signal was identified as toluene in the treatment. In addition, the retention time 3.6min when the peak appeared also corresponded well to such feature acquired above, as demonstrated in Fig. 7. The peak signal dropped from 5200pA to $3700 \mathrm{pA}$ that the area between the peak and axis decreased from 20600pA s to $14000 \mathrm{pA} s$ (after treatment). Toluene concentration after treatment was reduced to $230 \mathrm{ppm}$ which according to equation (2) that about $30 \%$ amount of the toluene was removed by the PDMS microparticles $(\eta \approx 30 \%)$. Although those spheres reached saturated status, total amount required to remove all the pollutants still remained to be investigated. Moreover, the absorption efficiency might be enhanced from perspectives such as increasing the amount, replacing as larger-sized particles, creating porous structure to enhance the specific surface area, adding additional functional groups to assist absorption, etc. Our recent experiment results had an improvement on the 
treatment efficiency to $45 \%$ by reducing the mass ratio of PDMS base and curing agent from 10:2 to 15:1. However, the reduction of curing agent made it more difficult to solidify the microdroplets to particles during thermal curing process. After treatment, the swelled PDMS were filtrated and the absorbed toluene by PDMS could be liberated simply by heating the PDMS particles. Thus, recovery and reuse of both PDMS and toluene could be realised. Other approach such as transferring the swelled microparticles into a lower soluble solvent might lead to a slower recovery and more time-consuming.

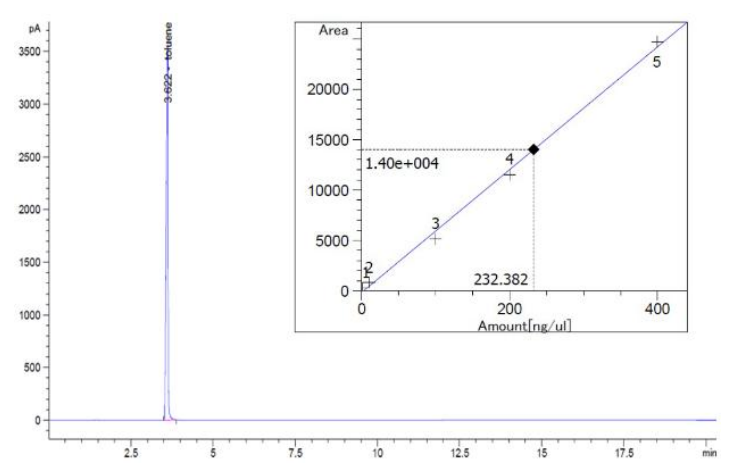

Fig. 7 Toluene peak signal after treatment by PDMS microspheres

\section{CONCLUSION}

We have fabricated PDMS microspheres by a needle-based microfluidic device based on Oil-in-Water single emulsion template. Flow rates of each phase can be tuned to produce microparticles with various diameters. The microparticles are highly monodispersed with the coefficient of variations (CV) for all groups being controlled under $1.5 \%$. A simulated organic wastewater that only contained toluene as an organic pollutant is treated by the PDMS microspheres. The results indicate that $30 \%$ of removing efficiency can be reached although there are a number of approaches to enhance the effect by for example applying larger particles or creating porous structures. The capability of PDMS microspheres for absorbing toluene and the great potential for scale-up by the needle-based microfluidic device provide great potential application towards removal of the organic pollutants in urban and industrial wastewater.

\section{ACKNOWLEDGMENTS}

This research was supported by Zhejiang Provincial Natural Science Foundation of China under Grant No. LQ15E090001, Young Scientist Program from National Natural Science Foundation of China under Grant No. NSFC51506103/E0605, and Ningbo Natural Science Foundation under Grant No. 2015A610281. The research was also supported by Inspiration Grant from Faculty of Science and Engineering, University of Nottingham Ningbo China. The author acknowledges the financial support from the International Doctoral Innovation Centre, Ningbo Education Bureau, Ningbo Science and Technology Bureau, and the University of Nottingham.

\section{REFERENCES}

1. Tiwari, D. K., Behari, J. \& Sen, P. Application of Nanoparticles in Waste Water Treatment. Carbon Nanotub. 3, 417-433 (2008).

2. Shannon, M. A. et al. Science and technology for water purification in the coming decades. Nat. (London, U. K.) 452, 301-310 (2008).

3. Dhote, J., Ingole, S. \& Chavhan, A. Review on Wastewater Treatment Technologies. Int. J. Eng. Res. Technol. 1, 1-10 (2012).

4. Miao, Z. \& Wang, L. The performance of starch microspheres treating wastewater. ICMREE2011 Proc. 2011 Int. Conf. Mater. Renew. Energy Environ. 1, 758-761 (2011).

5. Scott, A., Gupta, R. \& Kulkarni, G. U. A simple water-based synthesis of Au nanoparticle/PDMS composites for water purification and targeted drug release. Macromol. Chem. Phys. 211, 1640-1647 (2010).

6. Utada, A. S., Fernandez-Nieves, A., Stone, H. A. \& Weitz, D. A. Dripping to jetting transitions in coflowing liquid streams. Phys. Rev. Lett. 99, 1-4 
(2007).

7. Ottino, J. M. \& Wiggins, S. Introduction: mixing in microfluidics. Philos. Trans. R. Soc. London A 362, 923-935 (2004).

8. Abboud, J.-L. M. \& Notari, R. Critical compilation of scales of solvent parameters. Part I. Pure, non-hydrogen bond donor solvents. Pure Appl. Chem. 71, 645-718 (1999).

9. Lee, Park \& Whitesides. Solvent compatibility of poly (dimethylsiloxane)-based microfluidic devices. Anal. Chem. Dc- 75, 6544-6554 (2003).

10. Yoo, J. S., Kim, S. J. \& Choi, J. S. Swelling Equilibria of Mixed Solvent/Poly(dimethylsiloxane) Systems. $J$. Chem. Eng. Data 44, 16-22 (1999).

11. Koh, K. S., Chin, J., Chia, J. \& Chiang, C. L. Quantitative studies on PDMS-PDMS interface bonding with piranha solution and its swelling effect. Micromachines 3, 427-441 (2012).

12. Li, T., Zhao, L., Liu, W., Xu, J. \& Wang, J. Simple and reusable off-the-shelf microfluidic devices for the versatile generation of droplets. Lab Chip 4718-4724 (2016). doi:10.1039/C6LC00967K

13. Li, X., Li, F., Yang, J. \& Kinoshita, H. Study on the mechanism of droplet formation in T-junction microchannel. Chem. Eng. Sci. 69, 340-351 (2012).

14. Wu, L., Liu, X., Zhao, Y. \& Chen, Y. Role of local geometry on droplet formation in axisymmetric microfluidics. Chem. Eng. Sci. 163, 56-67 (2017).

15. Xu, J. H., Li, S. W., Tan, J. \& Luo, G. S. Correlations of droplet formation in T-junction microfluidic devices: From squeezing to dripping. Microfluid. Nanofluidics 5, 711-717 (2008).

16. Ward, T., Faivre, M. \& Stone, H. A. Drop production and tip-streaming phenomenon in a microfluidic flow-focusing device via an interfacial chemical reaction. Langmuir 26, 9233-9239 (2010).

17. Rotem, A., Abate, A. R., Utada, A. S., Van Steijn, V. \& Weitz, D. a. Drop formation in non-planar microfluidic devices. Lab Chip 12, 4263 (2012). 